\title{
Color doppler indices of proximal and distal parts of middle cerebral artery in fetuses with intrauterine growth restriction
}

Farhad Naleini ${ }^{1}$, Mohammad Farzizadeh ${ }^{1}$, Abdolmajid Taheri ${ }^{2}$, Ayoob Rostamzadeh ${ }^{3}$, Daryoush Fatehi ${ }^{4}$

${ }^{1}$ M.D., Assistant Professor of Radiology and Sonography, Faculty of Medicine, Kermanshah University of Medical Sciences, Kermanshah, Iran

${ }^{2}$ M.D., Assistant Professor of Radiology and Sonography, Shahrekord University of Medical Sciences, Shahrekord, Iran

${ }^{3}$ M.Sc. of Anatomical Sciences, Researcher in Cellular and Molecular Imaging, Shahrekord University of Medical Sciences, Shahrekord, Iran

${ }^{4}$ Ph.D., Associate Professor of Medical Physics, Faculty of Medicine, Shahrekord University of Medical Sciences, Shahrekord, Iran

\section{Type of article: Original}

\begin{abstract}
Introduction: Intrauterine growth restriction (IUGR) is a major clinical issue for pregnant women. The purpose of this study was to evaluate color Doppler indices of the proximal and distal parts of the middle cerebral artery (MCA) of the fetus.

Methods: In this cross-sectional study, 350 pregnant patients, with gestation age of 32-40 who were suspected to have intrauterine growth restriction, participated. The patients were referred for color Doppler sonography at the Imam Reza Hospital (Kermanshah, Iran) from May 2011 to September 2012. The following indices were measured for the proximal and distal part of the MCA: pulsatility index (PI), resistive index (RI), fetal heart rate (FHR), systolic to diastolic (S/D) ratio, and peak systolic velocity (PSV). The data were analyzed applying Tukey's-test, Paired-Samples t-test, and simple linear regression analysis using SPSS 19.

Results: Average age of the mother, the frequency of pregnancy, and fetus gestational age were $27.79 \pm 0.17$ years, $2.09 \pm 1.3$, and $34.19 \pm 2.52$ weeks, respectively. For gestation age of $<36 w e e k s$, all Doppler indices of the distal part of the fetus MCA were significantly different from those of proximal part $(p<0.05)$. Comparing indices of gestation age $<36$ weeks with those of $>36$ weeks, significant difference was found between the Doppler indices of the proximal parts as well as for the distal parts $(\mathrm{p}<0.05)$.

Conclusion: Measurement of fetus MCA indices may depend to the sampling location; however, this needs further investigation in order to find a clear probe location.

Keywords: Color Doppler, Intrauterine growth restriction, Middle cerebral artery, Pulse index, Resistance index
\end{abstract}

\section{Introduction}

Intrauterine growth restriction (IUGR) is a frequent clinical sign of chronic fetal hypoxemia. It is hard to distinguish between suboptimal fetal growth, because of intrauterine starvation and adequate growth of a constitutionally small infant, and it is generally prevalent in $3-10 \%$ of neonates (1). More common causes of IUGR include maternal, placental, and fetal factors. Factors related to the placenta in many cases of IUGR can include the placenta being too small and unable to provide sufficient nutrition to the growing baby e.g. lack of adequate oxygen supply to the fetus (2). In IUGR pregnancies, blood flow to the placenta decreases as pregnancy progresses, compared with normal pregnancy when blood flow to the placenta increases throughout pregnancy to meet the growing baby's demand for oxygen and nutrition (3). In cases of cell death (apoptosis) in pregnancies complicated by IUGR, the placenta contains a relatively high proportion of cells that have a shorter life than normal (4). Other causes of IUGR are

\section{Corresponding author:}

Daryoush Fatehi, Department of Medical Physics, Faculty of Medicine, Shahrekord University of Medical Sciences, Shahrekord, Iran. Tel: + 983833335652. Email: d.fatehi@gmail.com. Fax: +983813334911.

Received: September 27, 2016, Accepted: January 15, 2017, Published: May 2017

iThenticate screening: January 07, 2017, English editing: February 22, 2017, Quality control: March 28, 2017

(C) 2017 The Authors. This is an open access article under the terms of the Creative Commons Attribution-NonCommercialNoDerivs License, which permits use and distribution in any medium, provided the original work is properly cited, the use is non-commercial and no modifications or adaptations are made. 
factors related to the fetus including major congenital anomalies, trisomy 13,18, and 21, cytomegalovirus infection, and fetal alcohol syndrome $(2,5)$. Middle cerebral artery (MCA) velocimetry is a worthy sign of growth restricted fetus at risk of antenatal compromise (6). Detecting the fetus with pathological growth constraint that is at risk for perinatal problems is a current problem in obstetrics $(2,6)$. Most of the studies that report on IUGR have not distinguished the two terms of constitutionally and pathologically small fetuses (3, 7). Color Doppler Ultrasonography is a new method as a good predictor tool used for detecting abnormal blood flow in the vessels of fetus and mother, for example the uterine and placental artery, and also in assessment of patients with ovarian torsion $(6,8)$. In IUGR fetuses, there is a redistribution of the blood flow from the fetal periphery to the brain, moreover, Doppler studies of the circulation indicate that the human fetus responds to hypoxemia by centralizing blood flow to the brain, this phenomenon, the so called 'brain-sparing effect, is characterized by a decrease in fetal MCA pulsatility index (PI) (9). The MCA is a cerebral artery that has been studied frequently due to (a) good sampling, (b) providing data on the cerebral blood flow in normal and IUGR fetuses, and (c) it could be sampled at an angle of 0 ० between the ultrasound beam and the direction of blood flow $(6,10)$. The following parameters are measured to evaluate MCA: peak systolic velocity (PSV), end diastolic velocity (EDV), average velocity, PI, resistive index (RI), systolic to diastolic (S/D) ratio, and comparing parameters of MCA and umbilical artery (11). Arduini and Rizzo were the first to evaluate MCA of the normal or restricted fetuses with Doppler that is shown, although PI of the MCA in the restricted fetuses was not a suitable index for anticipation, but the ratio of MCA to umbilical artery was a better parameter (12). Veille JC. et al. found that with development of pregnancy, peak of the blood velocity, diameter and flow of the MCA increased, while the fetus heart total output toward the MCA had no change (13). Figueras F. et al. found that fetuses in the early stage of the pregnancy with abnormal PI of the MCA were weaker than those with normal PI of the MCA and umbilical artery (14). RI and PI can be measured in total length of the MCA, however, the problem is whether there is a difference for these indices in different parts of the MCA (6). Based on the brain sparing theory, in any form of fetus distress there is a reduction in blood flow which causes a decrease in brain arterial resistance in order to prevent brain ischemia (15). Since MCA acts as a reflector for left ventricle after load, several indices of the MCA, such as PI, RI, S/D, fetal heart rate (FHR), PSV, EDV, are measured in IUGR; however, PI, PSV, and RI are more clinically applicable $(12,14,16)$. In the present study, we measured the following indices: PI, RI, S/D, FHR, and PSV of the $1 / 3^{\text {rd }}$ proximal and the $1 / 3^{\text {rd }}$ distal of the MCA in the IUGR patients with $32^{\text {nd }}-40^{\text {th }}$ week of the gestation age. The purpose of the study was to find a relation between the indices and the location of the measurement as well as the gestational age.

\section{Material and Methods}

In a cross sectional and descriptive analytical study, 423 pregnant ladies participated randomly. This study was performed at the Imam Reza Hospital (Kermanshah, Iran) from May 2011 to September 2012. The patients were suspected to have IUGR. This study was approved by Research Ethics committee of Kermanshah University of Medical Sciences (Grant number 90233). A signed written consent was obtained from each patient. The study inclusion criteria were pregnancy between 32-40 weeks, and lack of known abnormalities in the fetus. Exclusion criteria were pregnancy with delivery problems including long delivery, pelvic inadaptability and pre-rupture of membrane (PROM). According to sonographic studies, the patients must suffer from IUGR to be qualified to enter the study. After obtaining their consent, Doppler Echography and velocimetry date including fetus MCA were registered. Gestational age was evaluated from day one of the previous menstrual period, and confirmed by a first or early second-trimester ultrasound scan in each patient. The ultrasound assessment of gestational age would be implemented if there was an inconsistency of 10 days or more between the first scan and the menstrual dates. Gestational age was confirmed by first-trimester ultrasound examination after it had initially been established by the last menstrual period. First-trimester ultrasound was used to calculate gestational age, when there was an inconsistency of more than 7 days. For study purposes, each of the following variables were recorded at antenatal test: (I) proximal MCAPI measured at the circle of Willis near the source of the MCA, (II) distal MCA-PI measured near the end of the greater wing of the sphenoid bone at the straight part of the artery. Doppler examinations were carried out by two sonologists with ten years' research experience in fetal Echography, using a G40 (Toshiba, Tokyo, Japan) ultrasound machine with the high-pass filter set at $50-100 \mathrm{~Hz}$, and a $3.5 \mathrm{MHz}$ convex probe with spatial peak temporal average intensity below $50 \mathrm{~mW} / \mathrm{cm}^{2}$. Each reading was carried out in the absence of gross fetal body or breathing movements and avoiding head compression by the transducer. For each examination, the mean value of three clear consecutive waveforms was recorded. The angle of insonation was permanently kept below $60^{\circ}$.

All patients were examined, and the fetus indices of PI, RI, FHR, S/D, and PSV at the $1 / 3^{\text {rd }}$ proximal and the $1 / 3^{\text {rd }}$ distal of the right and the left MCA were measured. In order to obtain desirable imaging from MCA, the following 
steps were performed for the patients suspected to have IURG. Firstly, we assessed the fetus head in transverse plane in which biometric measurement is performed, and after having the middle line, the image was taken in a manner that waves' direction was toward the fetus neck. Secondly, examination of the fetus head was performed in which the long axis was parallel to the probe, i.e. the angle was roughly $0^{\circ}$. In this step the fetus must be in resting time, having no motion for at least $2 \mathrm{~min}$. Thirdly, circle of Willis was imaged applying color Doppler and landmark of cerebral pons. In this step one must zoom in on the area of interest so that $50 \%$ of the screen shows the area illustrating the whole length of the MCA. As a technical point, one should notice that adjusted velocity settings of the color Doppler for circle of Willis arteries must be less than those for the umbilical artery. Fourthly, sample volume $(1 \mathrm{~mm})$ must be near the origin of the MCA of the internal carotid (in the proximal part of the MCA), in a manner that the angle between blood direction and beam direction is zero. In the evaluation of MCA, wave forms must be similar to each other to measure the highest PSV. In this study, we performed our measurement in this manner having the desirable PSV. To reduce error of the measurement, the steps of the study were repeated at least three times and the average of the values was recorded. The data was analyzed applying Paired-Samples T-test and Tukey's-test. SPSS software (Version 19; SPSS Inc., Chicago, USA) was used for the statistical analysis. The pvalues are two-sided at a significance level of $\leq 0.05$. A simple linear regression analysis was also performed to find correlation between the different Doppler indices.

\section{Results}

In total, of 423 women who were registered for the study, 73 were excluded, since they had no study criteria. Therefore, the data of 350 patients $(82.74 \%)$ was enrolled in the final analysis. The patients' average age, frequency of pregnancy, and average gestational age were $27.79 \pm 0.17$ years, $2.09 \pm 1.3$, and $34.19 \pm 2.52$ weeks, respectively. The results of the Doppler indices measured in the present study are summarized in Table 1. As Table 1 reveals, in patients with gestation age of less than 36 weeks, PI of the distal part of MCA was significantly higher than that of the proximal part ( $\mathrm{p}=0.009$ (. Moreover, a significant difference was found between the PI of the distal and proximal part in patients with gestation age greater than 36 weeks $(p=0.038)$. When we compared the data of the patients with gestation age less than 36 weeks with those of greater than 36 weeks, not only was there a significant different between the PI of the proximal parts $(p=0.007)$, but also, we found a significant different between the PI of the distal parts $(\mathrm{p}=0.012)$.

Table 1. Different color Doppler Echography indices obtained from proximal and distal parts of middle cerebral artery of the fetus with gestation age of less and greater than 36 weeks.

\begin{tabular}{|c|c|c|c|}
\hline \multirow{2}{*}{\multicolumn{2}{|c|}{ Doppler index }} & \multicolumn{2}{|l|}{ Gestational age (week) } \\
\hline & & $<36$ weeks $($ Mean $\pm \mathrm{SD})$ & $>36$ weeks $($ Mean $\pm \mathrm{SD})$ \\
\hline \multirow[t]{2}{*}{ PI (Pulsatility index) } & Proximal part & $1.24 \pm 0.16$ & $1.61 \pm 0.4$ \\
\hline & Distal part & $1.83 \pm 0.29^{\mathrm{a}}$ & $1.66 \pm 0.38^{c}$ \\
\hline \multirow[t]{2}{*}{ RI (Resistive index) } & Proximal part & $0.81 \pm 0.07$ & $0.8 \pm 0.06$ \\
\hline & Distal part & $0.88 \pm 0.05^{\mathrm{d}}$ & $0.81 \pm 0.06$ \\
\hline \multirow[t]{2}{*}{ FHR (Fetal heart rate) } & Proximal part & $150.69 \pm 5.88^{b}$ & $139.88 \pm 9.52$ \\
\hline & Distal part & $141.73 \pm 7.57$ & $134.71 \pm 7.76$ \\
\hline \multirow[t]{2}{*}{$\mathrm{S} / \mathrm{D}$ (Systolic to diastolic) ratio } & Proximal part & $6.19 \pm 1.37$ & $5.31 \pm 0.05$ \\
\hline & Distal part & $5.54 \pm 1.55$ & $3.16 \pm 0.44^{\mathrm{a}, \mathrm{c}}$ \\
\hline \multirow[t]{2}{*}{ PSV (Peak systolic velocity) } & Proximal part & $41.24 \pm 10.14^{\mathrm{b}}$ & $60.85 \pm 8.96^{\mathrm{d}}$ \\
\hline & Distal part & $23.15 \pm 2.69$ & $52.06 \pm 9.61^{\mathrm{c}}$ \\
\hline
\end{tabular}

SD: Standard Deviation; ${ }^{a} \mathrm{p}<0.05$ vs. proximal part in $<36$ weeks group; ${ }^{\mathrm{b}} \mathrm{p}<0.05$ vs. distal part in $<36$ weeks group; ${ }^{\mathrm{c}} \mathrm{p}<0.05$ vs. distal part in $<36$ weeks group; ${ }^{\mathrm{d}} \mathrm{p}<0.05$ vs. proximal part in $<36$ weeks group;

Table 1 demonstrates that in patients with gestation age of less than 36 weeks, RI of the distal part of MCA was not different from that of the proximal part $(\mathrm{p}=0.17)$. Furthermore, no difference was found between the RI of the distal and proximal part in patients with gestation age greater than 36 weeks $(p=0.22)$. When we compared the data of the patients with gestation age less than 36 weeks with those of greater than 36 weeks, we found a significant increase between the RI of the distal parts $(\mathrm{p}=0.026)$. As Table 1 illustrates, in patients with gestation age of less than 36 weeks, FHR of the distal part of MCA was significantly lower than that of the proximal part $(p=0.032)$. In contrast, no difference was found between the FHR of the distal and proximal part in patients with gestation age greater than 36 weeks $(p=0.092)$. When we compared the data of the patients with gestation age less than 36 weeks with those of greater than 36 weeks, not only was there a significant difference between the FHR of the proximal parts ( $\mathrm{p}=0.014)$, 
but also, we saw a significant difference between the FHR of the distal parts $(\mathrm{p}=0.037)$. Table 1 shows that in patients with gestation age of less than 36 weeks, S/D ratio of the distal part of MCA was no different than that of the proximal part $(\mathrm{p}=0.18)$. In contrast, a significant difference was found between the $S / D$ ratio of the distal and proximal part, in patients with gestation age greater than 36 weeks $(\mathrm{p}=0.023)$. Although, when we compared the data of the patients with gestation age less than 36 weeks with those of greater than 36 weeks, there was no significant difference between the $S / D$ ratio of the proximal parts $(p=0.097)$, but there was a significant difference between the $\mathrm{S} / \mathrm{D}$ ratio of the distal parts $(\mathrm{p}=0.011)$. As Table 1 shows, in patients with gestation age of less than 36 weeks, PSV of the distal part of MCA was significantly lower than that of proximal part $(\mathrm{p}=0.008)$. In contrast, there was no significant difference between the PSV of the distal and proximal part in patients with gestation age greater than 36 weeks $(p=0.18)$. When we compared the data of the patients with gestation age less than 36 weeks with those of greater than 36 weeks, not only was there a significant difference between the PSV of the proximal parts $(\mathrm{p}=0.019)$, but also, we saw a significant difference between the PSV of the distal parts $(\mathrm{p}=0.009)$. Additionally, the regression analysis showed that there was a strong positive linear correlation between RI and PI ( $p=0.036, r=0.783$ ), RI and $\mathrm{S} / \mathrm{D}$ ratio $(\mathrm{p}=0.031, \mathrm{r}=0.726)$, as well as PI and $\mathrm{S} / \mathrm{D}$ ratio $(\mathrm{p}=0.015, \mathrm{r}=0.754)$.

\section{Discussion}

In this study, we found that all Doppler indices of the distal part of fetus MCA were significantly different from those of the proximal part. The findings of the present study show that measurement of the MCA indices may depend on the location of sampling. The results of our study confirm the findings of other researchers. Figueras, Hsieh, and Locci independently showed that PI of the proximal part of MCA was remarkably lower than that of the distal part $(14,17,18)$. Hsieh also reported that RI obtained from three (proximal, middle, and distal) parts of the MCA observed significant difference. Hsieh showed that the average of PI to RI rate (PI/RI) of proximal, middle, and distal part of MCA were 1.61 to $0.82,1.77$ to 0.82 , and 1.84 to 0.84 respectively (17). Additionally, these researchers reported that PI of the proximal part was less than middle and distal parts. Also, significant reduction was found in PI/RI after 30 weeks of gestation, but the RI measured from the three parts of the MCA was not statistically different (17). Locci believed that variation in Doppler pattern of the proximal and distal parts of the MCA is due to the difference in the function of these two arteries' direction. Generally, these parts of the MCA are responsible for blood supply to the fetus brain, and they will develop during different stages of the fetal period, therefore, result in various patterns of the Doppler indices (18). Since blood velocity is affected on PI, we therefore believe that the difference of PI found in the present study for distal and proximal part is because of the difference between the blood velocities in these parts. Figueras and colleagues showed that in proximal part of the MCA, parameters such as PSV and peak EDV were higher than those of the distal part (14). Since there is a reverse relation between PI and blood velocity and increase in the velocity causes decrease in the blood impedance, reduction in the PI illustrates decrease of the impedance of blood flow. Therefore, regarding the findings of the present study, one can conclude that blood impedance of the proximal and distal parts of MCA are different, hence, PI is also different. Chang CC. et al. studied fetuses of 21 subjects with the 1st pregnancy in China in 2001. He found that the average of PI/RI of proximal, middle, and distal part of MCA were 1.62 to $0.80,1.69$ to 0.81 , and 1.71 to 0.83 , respectively, which were significantly different. The PI/RI was remarkably different for gestation phases: The PI/RI was 1.70 to 0.84 for $15-19$ weeks, 1.72 to 0.82 for $1520-24$ weeks, 1.68 to 0.83 for $25-29$ weeks, 1.65 to 0.81 for $30-34$ weeks, and 1.62 to 0.77 for $35-40$ weeks (19). Most of the scientists have investigated the proximal part of the MCA (20). Some of them studied the differences of the proximal and distal part (17). Bahlmann F. et al. (2002) evaluated 962 subjects with low risk pregnancy to find velocity of arterial blood in MCA in weeks 18 to 42 in a normal population. He found that maximum of the blood velocity continues to increase in this period $(\operatorname{Vmax}=26.8 \mathrm{~cm} / \mathrm{s}$ to $59.1 \mathrm{~cm} / \mathrm{s}$, Vmean $=11.2$ to $29.7 \mathrm{~cm} / \mathrm{s}$ and $\mathrm{Vmin}=4.9$ to $15.8 \mathrm{~cm} / \mathrm{s})(21)$. Clerici G. et al. (2002) has studied 20 ladies with the first pregnancy in weeks 36-40, to evaluate hemodynamic pattern of proximal and distal parts of MCA during movement and immovability of the fetus. He found that during fetus motion, there is remarkable reduction in the PI, stating decrease in the flow impedance in both proximal and distal parts of MCA ( 0.0028 vs. 0.00000085$)$. The average of fetus heart pulse rate during the immovability was remarkably lower than the motion phase $(\mathrm{p}=0.0047)$. However, there was no significant relation between PI of the proximal and distal parts of MCA in different behavior conditions of the fetus (22). Abel DE. et al. (2003) worked on 151 normal fetuses from weeks 16-42 to evaluate sonographic PVS of MCA. These researchers found a clear difference between the distal and proximal part of the MCA $(95 \% \mathrm{Cl}, 0.03-0.07$ and $0.07-0.12, \mathrm{P}<.001$ for both vessels), while there was no difference between the proximal parts $(95 \% \mathrm{Cl},-0.01$ to $0.03, \mathrm{p}=0.77)(23)$. Figueras (2004) prospectively evaluated 100 patients with first pregnancy in weeks 24-40 to find Doppler indices of the MCA in different locations for prognosis of umbilical cord gases in prolonged pregnancies. He found that there is a remarkable linear correlation between PI of the proximal and distal part $(\mathrm{R}=0.777 ; \mathrm{p}<0.0001)$, where the averages were $1.49 \pm 0.45$ and 
$1.56 \pm 0.47$, respectively. He also found a linear relation between the cerebroplacental ratios in the proximal and distal parts. Additionally, there was a remarkable relation between umbilical artery PO2, PI of proximal and distal parts of MCA (positive), and occurrence of elective Cesarean section (negative) (24). Clerici, who has worked in this field, could not find a sharp relation between PI and pulse rate of the fetus (22). We believe that although the present study presents valuable information, there is need to perform similar studies in other races and geographical areas, as well as patients with a history of metabolic and or vascular diseases, in order to find a better concept regarding the vascular changes in fetus. Authors assume that conducting a randomized study to decide the ideal timing of delivery of the growth-restricted fetus is too early, this is due to the fact that till now, no investigation has been able to distinguish different IUGR fetuses. Without these data, those investigations could not be of help, and confounding data could mislead the optimal timing for delivery of the growth restricted fetus. In this way, authors hesitate to introduce the best test possible and they think that before the full range of observational and randomized studies, deciding on the best test is unwise. This may complicate the history of considering a fetal heart rate monitoring in fetal surveillance. We believe that the study that we are doing could be the first step to knowing more about natural history of different IUGR fetuses.

\section{Conclusions}

Results of the present study showed that the Doppler indices of the proximal part of the middle cerebral artery of fetuses are different from those of the distal part. In conclusion, Doppler indices of MCA has been considered superior to those of other methods such as non-stress test, fetal biophysical profile and amniotic fluid index alone, in predicting adverse outcome in fetuses with IUGR. Regarding these findings, one can conclude that for measurement of these indices one must take notice to select the probe location, i.e. the proximal or distal part of the middle cerebral artery, as there are no equal responses from these places. However, still more investigations are needed to find a suitable probe location. Finally, PI of the fetal MCA is a key parameter

\section{Acknowledgments:}

This study was financially supported (grant no.: 90233) by the Deputy of Research at Kermanshah University of Medical Sciences, Kermanshah, Iran. The authors appreciate Dr. Mansour Rezaie for his statistical assistance.

\section{Conflict of Interest:}

There is no conflict of interest to be declared.

\section{Authors' contributions:}

All authors contributed to this project and article equally. All authors read and approved the final manuscript.

\section{References:}

1) Dhand H, Kansal HK, Dave A. Middle cerebral artery Doppler indices better predictor for fetal outcome in IUGR. J Obstet Gynaecol India. 2011; 61(2): 166-71. doi: 10.1007/s13224-011-0018-7. PMCID: PMC3394545.

2) Figueras F, Gardosi J. Intrauterine growth restriction: new concepts in antenatal surveillance, diagnosis, and management. Am J Obstet Gynecol. 2011; 204(4): 288-300. doi: 10.1016/j.ajog.2010.08.055. PMID: 21215383.

3) Fardiazar Z, Atashkhouei S, Yosefzad Y, Goldust M, Torab R. Comparison of fetal middle cerebral arteries, umbilical and uterin artery color Doppler ultrasound with blood gas analysis in pregnancy complicated by IUGR. Iran J Reprod Med. 2013; 11(1): 47-51. PMID: 24639692, PMCID: PMC3941376.

4) Scifres CM, Nelson DM. Intrauterine growth restriction, human placental development and trophoblast cell death. J Physiol. 2009; 587(Pt 14): 3453-8. doi: 10.1113/jphysiol.2009.173252. PMID: 19451203, PMCID: PMC2742274.

5) Resnik R. Intrauterine growth restriction. Obstet Gynecol. 2002; 99(3): 490-6. doi: 10.1097/00006250200203000-00020. PMID: 11864679.

6) Khanduri S, Parashari UC, Bashir S, Bhadury S, Bansal A. Comparison of diagnostic efficacy of umbilical artery and middle cerebral artery waveform with color Doppler study for detection of intrauterine growth restriction. J Obstet Gynaecol India. 2013; 63(4): 249-55. doi: 10.1007/s13224-012-0326-6. PMID: 24431651, PMCID: PMC3763050.

7) Faraci M, Renda E, Monte S, Di Prima FA, Valenti O, De Domenico R, et al. Fetal growth restriction: current perspectives. J Prenat Med. 2011; 5(2): 31-3. PMID: 22439073 , PMCID: PMC3279162. 
8) Rostamzadeh A, Mirfendereski S, Rezaie MJ, Rezaei S. Diagnostic efficacy of sonography for diagnosis of ovarian torsion. Pak J Med Sci. 2014; 30(2): 413-6. PMID: 24772154 , PMCID: PMC3999021.

9) Bano S, Chaudhary V, Pande S, Mehta V, Sharma A. Color doppler evaluation of cerebral-umbilical pulsatility ratio and its usefulness in the diagnosis of intrauterine growth retardation and prediction of adverse perinatal outcome. Indian J Radiol Imaging. 2010; 20(1): 20-5. doi: 10.4103/0971-3026.59747. PMID: 20351987, PMCID: PMC2844742.

10) Mari G, Hanif F. Fetal Doppler: umbilical artery, middle cerebral artery, and venous system. Semin Perinatol. 2008; 32(4): 253-7. doi: 10.1053/j.semperi.2008.04.007. PMID: 18652923.

11) Tarzamni MK, Nezami N, Gatreh-Samani F, Vahedinia S, Tarzamni M. Doppler waveform indices of fetal middle cerebral artery in normal 20 to 40 weeks pregnancies. Arch Iran Med. 2009; 12(1): 29-34. PMID: 19111026.

12) Arduini D, Rizzo G, Romanini C. Changes of pulsatility index from fetal vessels preceding the onset of late decelerations in growth-retarded fetuses. Obstet Gynecol. 1992; 79(4): 605-10. PMID: 1553186.

13) Veille JC, Hanson R, Tatum K. Longitudinal quantitation of middle cerebral artery blood flow in normal human fetuses. Am J Obstet Gynecol. 1993; 169(6): 1393-8. PMID: 8267034.

14) Figueras F, Fernandez S, Eixarch E, Gomez O, Martinez JM, Puerto B, et al. Middle cerebral artery pulsatility index: reliability at different sampling sites. Ultrasound Obstet Gynecol. 2006; 28(6): 809-13. doi: 10.1002/uog.2816. PMID: 17019746.

15) Cohen E, Baerts W, Van Bel F. Brain-Sparing in Intrauterine Growth Restriction: Considerations for the Neonatologist. Neonatology. 2015; 108(4): 269-76. doi: 10.1159/000438451. PMID: 26330337.

16) Mari G, Hanif F, Kruger M, Cosmi E, Santolaya-Forgas J, Treadwell MC. Middle cerebral artery peak systolic velocity: a new Doppler parameter in the assessment of growth-restricted fetuses. Ultrasound Obstet Gynecol. 2007; 29(3): 310-6. doi: 10.1002/uog.3953. PMID: 17318946.

17) Hsieh YY, Chang CC, Tsai HD, Tsai CH. Longitudinal survey of blood flow at three different locations in the middle cerebral artery in normal fetuses. Ultrasound Obstet Gynecol. 2001; 17(2): 125-8. doi: 10.1046/j.1469-0705.2001.00329.x. PMID: 11251920.

18) Locci M, Nazzaro G, De Placido G, Montemagno U. Fetal cerebral haemodynamic adaptation: a progressive mechanism? Pulsed and color Doppler evaluation. J Perinat Med. 1992; 20(5): 337-43. PMID: 1479515.

19) Chang CC, Hsieh YY, Tsai HD. Doppler study of the fetal middle cerebral artery at three locations: preliminary report. Chang Gung Med J. 2001; 24(7): 418-22. PMID: 11565247.

20) Shono M, Shono H, Ito Y, Muro M, Uchiyama A, Sugimori H. The effect of behavioral states on fetal heart rate and middle cerebral artery flow-velocity waveforms in normal full-term fetuses. Int J Gynaecol Obstet. 1997; 58(3): 275-80. PMID: 9286860.

21) Bahlmann F, Reinhard I, Krummenauer F, Neubert S, Macchiella D, Wellek S. Blood flow velocity waveforms of the fetal middle cerebral artery in a normal population: reference values from 18 weeks to 42 weeks of gestation. J Perinat Med. 2002; 30(6): 490-501. doi: 10.1515/JPM.2002.077. PMID: 12530106.

22) Clerici G, Luzietti R, Cutuli A, Direnzo GC. Cerebral hemodynamics and fetal behavioral states. Ultrasound Obstet Gynecol. 2002; 19(4): 340-3. doi: 10.1046/j.1469-0705.2002.00634.x. PMID: 11952961.

23) Abel DE, Grambow SC, Brancazio LR, Hertzberg BS. Ultrasound assessment of the fetal middle cerebral artery peak systolic velocity: A comparison of the near-field versus far-field vessel. Am J Obstet Gynecol. 2003; 189(4): 986-9. PMID: 14586340.

24) Figueras F, Lanna M, Palacio M, Zamora L, Puerto B, Coll O, et al. Middle cerebral artery Doppler indices at different sites: prediction of umbilical cord gases in prolonged pregnancies. Ultrasound Obstet Gynecol. 2004; 24(5): 529-33. doi: 10.1002/uog.1738. PMID: 15459935. 